九州大学学術情報リポジトリ

Kyushu University Institutional Repository

\title{
ON BERRY-ESSEEN THEOREM FOR NONPARAMETRIC DENSITY ESTIMATION IN MARKOV SEQUENCES
}

Basu, A. K.

Calcutta University

Sahoo, D. K.

Calcutta University

https://doi.org/10.5109/13467

出版情報: Bulletin of informatics and cybernetics. 30 (1)，pp.25-39，1998-03. Research Association of Statistical Sciences

バージョン:

権利関係 : 


\title{
ON BERRY-ESSÉEN THEOREM FOR NONPARAMETRIC DENSITY ESTIMATION IN MARKOV SEQUENCES
}

\author{
By
}

\author{
A.K. BASU ${ }^{*}$ and D.K. SAHoo ${ }^{\dagger}$
}

\begin{abstract}
For a stationary sequence $\left\{X_{i}\right\}$ the Markov assumption $G_{2}$, which is weaker than the Doeblin's condition $D_{0}$, is discussed and is used to estimate nonparametric density and transition density. Under the $G_{2-}$ assumptions, the rate of convergence to normality of the estimated density is derived. Similar type of results are also derived for estimating the joint density and the estimated transition density.
\end{abstract}

Key words : Nonparametric density estimation, Berry-Esséen theorem, $G_{2}$-Markov sequence.

AMS Subject Classification No. : Primary - 62G07, Secondary - 60F05.

\section{Introduction}

Non parametric estimators of the density function of a population based on a sample of independent observations have been considered by several authors. An excellent survey of the results in this area is given by Rosenblatt (1971). Some authors also consider the estimates of the density function when the observations are dependent in the sense that they are sampled from a stationary Markov sequence. Roussas (1967, 1969) considers density estimation from Markov processes and obtained consistency and asymptotic normality of Kernel type density estimators, under the Doeblin's condition $D_{0}$ as given in Doob (1953).

In this paper we consider Markov assumption $G_{2}$ which is discussed and employed by Rosenblatt (1970) and Yakowitz $(1985,1989)$ and others.

A stationary Markov sequence $\left\{X_{i}\right\}$ is a $G_{2}$-sequence if there is a positive number $\rho<1$ and a positive integer $n$ such that for any bounded Borel function $h(\cdot)$ satisfying $E\left[h\left(X_{i}\right)\right]=0$, we have

$$
E_{X_{1}}\left[E\left(h^{2}\left(X_{n}\right) \mid X_{1}\right)\right] \leq \rho^{2} E\left[h^{2}\left(X_{1}\right)\right]
$$

This assumption is weaker than $D_{0}$. In fact Rosenblatt (1970) considered an example where $D_{0}$ is not satisfied but $G_{2}$ holds. He also considered Kernel type density estimators

\footnotetext{
* Calcutta University

$\dagger$ R.K. Mission College, Narendrapur
} 
when the observations are sampled from a stationary Markov process and observed that these estimators have the same character as in the case of independence. He proved the asymptotic normality of the Kernel type estimators but does not find out the rate of convergence. In this paper our aim is to obtain Berry-Esséen type bound of the Kernel type density estimator under the Markov assumption $G_{2}$. Yakowitz and his colleagues (1985) while analysing river flow data found that non-parametric model suits more than the popular autoregressive-moving-avergae (ARMA) model. He also found that under some restrictions ARMA model can be transformed into two-dimensional $G_{2}$-Markov sequence. In fact it is clear from the discussion of Rosenblatt (1970) and Yakowitz (1985) that Markov assumption does not play significant role but stationarity and $G_{2}$-condition make the proofs of our results valid and moreover the conclusion of our results is true if the first component of a two-dimensional Markov sequence satisfies $G_{2}$-condition. Under $G_{2}$-assumption we also obtain rate of convergence to normality for estimated joint density and transition density. In this connection it is to be noted that Prakasa Rao (1977) derived the Berry-Esséen bound for estimating only the density under stronger Doeblin's condition. Berry-Esséen bounds are also obtained by Basu and Sahoo (1988, 1989) for density estimates under different conditions and set up. In Section 2 we state the Markov assumption and consider the method for estimating the unknown stationary density. Using the methodology of Section 3 asymptotic normality together with rate of convergence of the Section 3 and Section 4 are used to prove the corresponding results for the estimate of the transition density in Section 5. Last of all some concluding remarks are added to Section 6 . Sometimes we use the Vinogradov symbol $<<$ to indicate an inequality containing some unspecified positive constant factor.

\section{The Markov Assumption and the Estimation of the density}

Let $\left\{X_{i}\right\}$ be a stationary Markov sequence with a continuous stationary density function $f(x)>0$ and a continuous transition p.d.f. $f(y / x)$. Let $f_{j-i}(y, x)$ and $f_{j-i}(y \mid x)$ denote the joint probability density of $X_{j}$ and $X_{i}$ and the conditional probability density of $X_{j}$ given $X_{i}$ respectively, for $i \neq j$.

Obviously then,

$$
f_{j-i}(y, x)=f_{j-i}(y \mid x) \cdot f(x)
$$

Define

$$
\hat{f}_{n}(x)=(n h)^{-1} \sum_{j=1}^{n} K\left(\left(x-X_{j}\right) / h\right)
$$

as the estimate of $f(x)$ where

$$
\text { i) } h=h(n)>0 \text { are such that } h(n) \rightarrow 0 \text { and } n h(n) \rightarrow \infty \text { as } n \rightarrow \infty
$$

ii) $K(\cdot)$ is a given bounded continuous density function such that

$$
\begin{gathered}
K(-u)=K(u), \int u K(u) d u=0, \int u^{i} k^{j}(u) d u<\infty \\
\text { for } i=0,1,2 ; j=1,2,3,
\end{gathered}
$$


and iii) $f(x)$ is continuously differentiable upto 2 nd order.

We note that $\hat{f}_{n}(x)$ is the same sort of estimate of $f(x)$ as in the case of independent observations with

$V\left(\hat{f}_{n}(x)\right)=(n h)^{-1} f(x) \int K^{2}(x) d x+O\left(\frac{1}{n}\right)$. (by computation and also see Rosenblatt (1970)).

\section{Speed of Convergence to Asymptotic normality of the estimated density}

Consider $W_{n}=(n h)^{1 / 2}\left[\hat{f}_{n}(x)-E \hat{f}_{n}(x)\right] /\left[f(x) \int K^{2}(x) d x\right]^{1 / 2}$

$$
=\sum_{j=1}^{n}(n h)^{-1 / 2}\left[K\left(\left(x-X_{j}\right) / h\right)-E K\left(\left(x-X_{j}\right) / h\right)\right] /\left(f(x) \int K^{2}(x) d x\right)^{1 / 2} .
$$

The asymptotic normality and its speed of convergence is obtained by writing the sum as a sum of big blocks separated by small blocks where the big blocks are approximately independent and then applying a well known lemma for the sum of two sequences of random variables which is stated below :

Lemma 3.1. Let $W_{n}=Z_{n}+Y_{n}, n=1,2, \ldots$ where $\left\{W_{n}\right\}_{n \geq 1}$ and $\left\{Z_{n}\right\}_{n \geq 1}$ are sequences of random variables. Let $\psi$ be a distribution function with a bounded derivative. Suppose $\left\{b_{n}\right\}_{n \geq 1}$ is a sequence of positive constants satisfying

$$
\sup _{t}\left|P\left[Z_{n} \leq t\right]-\psi(t)\right|=O\left(b_{n}\right), n \rightarrow \infty
$$

Then for any sequence of positive constants $\left\{a_{n}\right\}_{n \geq 1}$ we have

$$
\sup _{t}\left|P\left[W_{n} \leq t\right]-\psi(t)\right|=O\left(b_{n}\right)+O\left(a_{n}\right)+P\left[\left|Y_{n}\right|>a_{n}\right], n \rightarrow \infty .
$$

Write

$$
W_{n}=\sum_{1}^{k}\left(A_{l}+B_{l}\right)+H
$$

where

$$
\begin{aligned}
& A_{l}=\sum_{j=(l-1)(m+r)+1}^{l m+(l-1) r} T_{n j} /\left(f(x) \int K^{2}(x) d x\right)^{1 / 2} \\
& B_{l}=\sum_{j=l(m+r)}^{l m+(l-1) r+1} T_{n j} /\left(f(x) \int K^{2}(x) d x\right)^{1 / 2} \\
& H=\sum_{j=k(m+r)+1}^{n} T_{n j} /\left(f(x) \int K^{2}(x) d x\right)^{1 / 2}
\end{aligned}
$$

and $T_{n j}=(n h)^{-1 / 2}\left[K\left(\left(x-X_{j}\right) / h\right)-E\left\{K\left(\left(x-X_{j}\right) / h\right)\right\}\right]$.

Here $A_{l}$ and $B_{l}$ are big and small blocks respectively with $m=m(n) \rightarrow \infty$ and $r=r(n) \rightarrow \infty$ as $n \rightarrow \infty$ but $m(n)=o(n)$ and $r(n)=o(m(n))$. 
Further $k=k(n)=[n /(m+r)]$, the greatest integer containing $n /(m+r)$, tends to infinity as $n \rightarrow \infty$, since $m, r=o(n)$. Here $H$ accounts for the additional few terms at the end not included in big blocks or small blocks.

Assumption: We select $m, h, k$ and $r$ such that

$$
\begin{aligned}
& m(n)=m=O\left(n^{7 / 10}\right) \\
& h(n)=h=O\left(n^{-1 / 5}\right) \\
& k(n)=k=O\left(n^{3 / 10}\right) \\
& r(n)=r<O\left(n^{1 / 5}\right) \text { such that } r=o(m)
\end{aligned}
$$

We write $W_{n}$ as $W_{n}=Z_{n}+Y_{n}$ where $Z_{n}=\sum_{1}^{k} A_{l}$ and $Y_{n}=\sum_{1}^{k} B_{l}+H$.

Here $A_{l}$ are treated as independent variables with the same marginal distributions. Also $E\left(A_{l}\right)=0$ and $S_{n}^{2}=V\left(\sum_{1}^{k} A_{l}\right)=o(n h)$ (see Rosenblatt (1970)). Now we have the following Theorem.

THEOREM 3.1. Let $\Phi$ be the distribution function of the univariate standard normal distribution then under the assumption (3.6)

$$
\sup _{t}\left|P\left[Z_{n} \leq t\right]-\Phi(t)\right|=O\left(n^{-1 / 5}\right) .
$$

Proof. Under (3.6)

$$
\left.S_{n}^{2}=V\left(\sum_{1}^{k} A_{l}\right)<<n^{4 / 5}\right) .
$$

Now by repeated application of $C_{r}$-inequality we have,

$$
\begin{aligned}
E\left|A_{l}\right|^{3}= & (n h)^{-3 / 2}\left\{f(x) \int K^{2}(x) d x\right\}^{-3 / 2} E \mid \sum_{j=(l-1)(m+r)+1}^{l m+(l-1) r}\left[K\left(\left(x-X_{j}\right) / h\right)\right. \\
& \left.-E K\left(\left(x-X_{j}\right) / h\right)\right]\left.\right|^{3}<<m^{2}(n h)^{-3 / 2} \sum_{j}\left[E K^{3}\left(\left(x-X_{j}\right) / h\right)\right. \\
& \left.+E^{3} K\left(\left(x-X_{j}\right) / h\right)\right] \\
= & m^{2}(n h)^{-3 / 2} \sum_{j}\left[h \int_{-\infty}^{\infty} K^{3}(u) f(x-h u) d u+\left\{h \int_{-\infty}^{\infty} K(u) f(x-h u) d u\right\}^{3}\right] \\
= & m^{2}(n h)^{-3 / 2} \sum_{j}\left[O\left(h^{3}\right)+h f(x) \int_{-\infty}^{\infty} K^{3}(u) d u+\left\{h f(x)+O\left(h^{3}\right)\right\}^{3}\right] \\
\quad \text { by Taylor's expansion and using }(2.3),(2.4) \text { and }(2.5) & O\left(m^{3}\left(n^{3} h\right)^{-1 / 2}\right) .
\end{aligned}
$$

This implies

$$
\begin{aligned}
\sum_{l=1}^{k} E\left|A_{l}\right|^{3} & =O\left(k m^{3} n^{-3 / 2} h^{-1 / 2}\right) \\
& =O(n) \text { under }(3.6)
\end{aligned}
$$


Hence by Berry-Esséen theorem for independent random variables we get

$$
\sup _{t}\left|P\left[\sum_{1}^{k} A_{l} \leq t\right]-\Phi(t)\right| \leq \text { const. } \sum_{l=1}^{k} E\left|A_{l}\right|^{3} / s_{n}^{3}
$$

Therefore $\sup _{t}\left|P\left[\sum_{1}^{k} A_{l} \leq t\right]-\Phi(t)\right|=O\left(n^{-1 / 5}\right)$ using (3.8) and (3.9) i.e.

$$
\sup _{t}\left|P\left[Z_{n} \leq t\right]-\Phi(t)\right|=O\left(n^{-1 / 5}\right) .
$$

Hence the Theorem 3.1 is proved.

In order to get an estimate of $\sup _{x}\left|P\left[\sum_{1}^{k} A_{l} \leq x\right]-\Phi(x)\right|$ we have to consider the characteristic function of $\sum_{1}^{k} A_{l}$ and to compare this characteristic function with the product of the characteristic functions of the individual $A_{l}$ 's. Following Rosenblatt (1970, p. 207) we find that

$$
\left|E\left\{\exp \left(i t \sum_{1}^{k} A_{l}\right)\right\}-\pi_{1}^{k} E\left\{\exp \left(i t A_{l}\right)\right\}\right| \leq(k-1) M \rho^{r(n)} \rightarrow 0
$$

where $M$ is a constant and $0<\rho<1$ and also $k(n) \rho^{r(n)} \rightarrow 0$.

Now from basic inequality (Loeve) and Esséen's lemma we have,

$$
\begin{aligned}
& \left|P\left[\sum_{1}^{k} A_{l} \leq x\right]-\Phi(x)\right| \leq\left|P\left[\sum_{1}^{k} A_{l} \leq x\right]-G(x)\right|+|G(x)-\Phi(x)| \\
& \leq \frac{2}{\pi} \int_{0}^{u} t^{-1}\left|E\left[e^{i t} \sum_{1}^{k} A_{l}\right]-\sum_{l=1}^{k} E\left(e^{i t A_{l}}\right)\right| d t+\frac{24}{\pi u} \sup _{x} G^{\prime}(x)+O\left(n^{-1 / 5}\right)
\end{aligned}
$$

where $u>0$ and $G(x)$ is the distribution function of $\sum_{1}^{k} A_{l}$ when $A_{l}$ are treated as independent but with same marginal distributions as defined in (3.5).

$$
\leq \frac{2}{\pi}(k-1) M \rho^{r(n)} \log |u|+\frac{24}{\pi u} \sup _{x} G^{\prime}(x)+O\left(n^{-1 / 5}\right) .
$$

For a given $0<\rho<1$, selecting a suitable constant satisfying $r=0(\log n)$ and selecting $u=O\left(n^{1 / 5}\right)$ and assuming $\sum_{1}^{k} A_{l}$ has finite density at $x$,

$$
\begin{aligned}
\left|P\left[\sum_{1}^{k} A_{l} \leq x\right]-\Phi(x)\right| & =O\left(n^{-1 / 5} \log n\right)+O\left(n^{-1 / 5}\right) \\
& =O\left(n^{-1 / 5} \log n\right)
\end{aligned}
$$


We can represetn $Y_{n}$ in the following form -

$$
\begin{gathered}
Y_{n}=\sum_{l=1}^{k} B_{l}+H \\
=\frac{\sum_{j=m+1}^{m+r} T_{n j}+\sum_{j=2 m+r+1}^{2(m+r)} T_{n j}+\ldots+\sum_{j=k m+(k-1) r+1}^{k(m+r)} T_{j n}+\sum_{j=k(m+r)+1}^{n} T_{n j}}{\left(f(x) \int K^{2}(x) d x\right)^{1 / 2}} \\
=\left[\sum_{j \leq k+1} \chi_{n j}\right] \begin{array}{l}
/\left(f(x) \int K^{2}(x) d x\right)^{1 / 2} \text { where } \chi_{n 1}=T_{n m+1}+\ldots+T_{n m+r} \\
\chi_{n k}=T_{n k m+\overline{k-1} r+1}+\ldots+T_{n k(m+r)} \\
\chi_{n k+1}=T_{n k(m+r)+1}+\ldots+T_{n n} .
\end{array}
\end{gathered}
$$

under this representation and also under assumption (3.6) we have the following theorem:

THEOREM 3.2 .

$$
P\left[\left|Y_{n}\right| \geq \varepsilon\right]=O\left(n^{-1}\right)
$$

where $\varepsilon=O\left(n^{-1 / 4}(\log n)^{3}\right)$.

Proof. Since $\left\|\chi_{n j}\right\|_{\infty}=O\left(r(n) n^{-2 / 5}\right)=c_{n}$, we have for $i \leq j \leq k+1$ and $O<t<c_{n}^{-1}$, following Philipp (1969)

$$
E\left(e^{t \chi_{n j}}\right)<e^{t^{2} c_{n}^{2}} .
$$

Also following (4.1) of Rosenblatt (1970)

$$
E\left|e^{t Y_{n}}-\pi_{j}\left(E e^{t \chi_{n j}}\right)\right| \leq k M \rho^{r(n)}
$$

where $M$ is a constant and $O<\rho<1$.

Hence it follows from (3.6) and (3.15) that

$$
E\left(e^{t Y_{n}}\right)<<\underset{j \leq k+1}{\pi} E\left(e^{t \chi_{n j}}\right)<<e^{t^{2} c_{n}^{2}(k+1)} .
$$

To conclude the proof we state the following well known result (without proof).

RESULT 3.1. If $\left\{V_{n}\right\}$ is a sequence of random variables such that $E\left(e^{t V_{n}}\right)$ exists for some $t>0$ then

$$
P\left[\left|V_{n}\right| \geq \varepsilon\right] \leq E\left(e^{t V_{n}}\right) / e^{t \varepsilon} \text { for all } \varepsilon>0
$$

Now setting $t=$ const. $n^{1 / 4} \log n, \varepsilon=$ const. $n^{-1 / 4}(\log n)^{3}$ and using (3.6) and (3.17) on the Result 3.1 we get the R.H.S. of (3.14) by choosing $r(n)=O(\log n)$ so that $r(n)<O\left(n^{1 / 5}\right)$ is satisfied.

Hence the Theorem 3.2 . 
Theorem 3.3 Rate of Convergence to normality. If $\Phi$ be the distribution function of the univariate standard normal distribution and $x$ be a point of continuity of $f$ then under assumption (3.6) with $r(n)=O(\log n)$

$$
\sup _{t}\left|P\left[\left(\hat{f}_{n}(x)-E \hat{f}_{n}(x)\right) /\left(\sqrt{V}\left[\hat{f}_{n}(x)\right]\right) \leq t\right]-\Phi(t)\right|=O\left(n^{-1 / 5}\right) .
$$

Proof. Note that, by (2.6) for $0<u \geq O\left(n^{3 / 5}\right), P\left(\mid \sqrt{n h V\left(\hat{f}_{n}(x)\right) / f(x) \int K^{2}(x) d x}\right.$ $-1 \mid>u)=0$ as $n \rightarrow \infty$. Selecting $b_{n}=n^{-1 / 5}, a_{n}=\varepsilon=0\left(n^{-1 / 4}(\log n)^{3}\right)$, taking $\psi$ to be $\Phi$ and lastly applying the result of Theorem 3.1 and Theorem 3.2 on lemma 3.1 and on the well known result of Michel and Pfanzagl (1971) we have,

$$
\sup _{t}\left|P\left[W_{n} \leq t\right]-\Phi(t)\right|=O\left(n^{-1 / 5}\right)+O\left(n^{-1 / 4}(\log n)^{3}\right)+O\left(n^{-1}\right)
$$

Therefore, $\sup _{t}\left|P\left[\left\{\left(\hat{f}_{n}-E\left(\hat{f}_{n}\right)\right) / \sqrt{V}\left[\hat{f}_{n}\right]\right\} \leq t\right]-\Phi(t)\right|=O\left(n^{-1 / 5}\right)$

[proved]

\section{Speed of Convergence to normality of the estimated stationary density}

Let $f(x, y)$ be the stationary probability density function of $\left(X_{j}, X_{j+1}\right)$. We assume that all joint distributions with a finite number of distinct $X_{j}$ 's are absolutely continuous with uniformly bounded continuous density functions. A natural estimator for the stationary probability is therefore,

$$
\hat{f}_{n}(x, y)=\left(n h^{2}\right)^{-1} \sum_{j=1}^{n-1} K\left(\left(x-X_{j}\right) / h,\left(y-X_{j+1}\right) / h\right)
$$

where $K(x, y)$ is a bounded continuous density function satisfying $\int u^{2} K(u, v) d u d v<\infty$ and $\int v^{2} K(u, v) d u d v<\infty$. Note that $V\left(\hat{f}_{n}(x, y)\right)=\left(n h^{2}\right)^{-1} f(x, y) \int K^{2}(x, y) d x d y$ $+O(1 / n h)$. Choosing $m(n)=O\left(n^{18 / 35}\right), k(n)=O\left(n^{17 / 35}\right), r(n)=O(\log n)$ and following exactly similar procedure as in Section 3 we have the following theorem.

THEOREM 4.1. If $\Phi$ be the distribution function of standard normal distribution and $(x, y)$ be a point of continuity of ' $f$ ' then

$$
\left.\sup _{t} \mid P\left[\left\{\hat{f}_{n}(x, y)-E \hat{f}_{n}(x, y)\right) /\left(V\left[\hat{f}_{n}(x, y)\right]\right)^{1 / 2}\right\} \leq t\right]-\Phi(t) \mid=O\left(n^{-1 / 7}(\log n)^{3}\right)
$$

where $h=O\left(n^{-1 / 5}\right)$.

To reduce the bias term in Theorem 4.2 and Theorem 5.2 we assume well known bias reduction criterion $\iint u^{i} v^{j} K(u, v) d u d v=0$ for $i, j=0,1$ and $i+j \neq 0$.

THEOREM 4.2. Under the conditions of Theorem 4.1 and $h_{n}=n^{-\alpha}, \alpha=\frac{1}{5}$,

$$
\sup _{t}\left|P\left[\frac{\hat{f}_{n}(x, y)-f(x, y)}{\sqrt{V} \operatorname{ar}\left\{\hat{f}_{n}(x, y)\right\}} \leq t\right]-\Phi(t)\right|=O\left(n^{-\frac{1}{10}+\tau}\right) ; 0<\tau<\frac{1}{10}
$$

provided the partial derivatives of $f(x, y)$ upto 2 nd order are continuous and 3 rd order derivatives are finite. 
Proof. We know that

$$
\begin{aligned}
E \hat{f}_{n}(x, y) & =\frac{1}{n h^{2}} \sum_{j=1}^{n-1} \iint K\left[\left(x-x_{j}\right) / h,\left(y-x_{j+1}\right) / h\right] f\left(x_{j}, x_{j+1}\right) d x_{j} d x_{j+1} \\
& =\frac{n-1}{n h^{2}} \iint K\left[\left(x-x_{1}\right) / h,\left(y-y_{1}\right) / h\right] f\left(x_{1}, y_{1}\right) d x_{1} d y_{1}
\end{aligned}
$$

Using a change of variable and Taylor's expansion of $f(x, y)$ yields

$$
\begin{aligned}
E\left(\hat{f}_{n}(x, y)\right)= & \frac{n-1}{n} \iint K(u, v) f(x-u h, y-v h) d u d v \\
= & \frac{n-1}{n} \iint K(u, v)\left[f(x, y)-h u f_{x}(x, y)-h v f_{y}(x, y)\right. \\
& \left.+\frac{h^{2} u^{2}}{2} f_{X X}(x, y)+\frac{h^{2} v^{2}}{2} f_{Y Y}(x, y)+h^{2} u v f_{X Y}(x, y)+O\left(h^{3}\right)\right] d u d v \\
= & f(x, y)+O\left(h^{2}\right)
\end{aligned}
$$

Therefore,

$$
\left|E \hat{f}_{n}(x, y)-f(x, y)\right|=O\left(h^{2}\right)=O\left(n^{-2 \alpha}\right)
$$

Also,

$$
\operatorname{Var}\left[\hat{f}_{n}(x, y)\right]=O\left(\frac{1}{n h^{2}}\right)=O\left(n^{-1(1-2 \alpha)}\right)
$$

Define

$$
\frac{\hat{f}_{n}(x, y)-f(x, y)}{\sqrt{V} a r\left\{\hat{f}_{n}(x, y)\right\}}=Z_{n}^{*}+Y_{n}^{*}
$$

where

$$
Z_{n}^{*}=\frac{\hat{f}_{n}(x, y)-E \hat{f}_{n}(x, y)}{\sqrt{\operatorname{V} a r}\left(\hat{f}_{n}(x, y)\right)} \text { and } Y_{n}^{*}=\frac{E \hat{f}_{n}(x, y)-f(x, y)}{\sqrt{V} \operatorname{ar}\left(f_{n}(x, y)\right)}
$$

Using (4.4) and (4.5) we see that

$$
\left|Y_{n}^{*}\right|=O\left(\frac{n^{-2 \alpha}}{n^{-(1-2 \alpha) / 2}}\right)=O\left(n^{\frac{1-6 \alpha}{2}}\right) .
$$

We select ' $a$ ' such that $n^{-a}>n^{\frac{1-6 \alpha}{2}}$ (i.e. ' $a$ ' is selected such that $6 \alpha-2 a-1>0$ ) and then

$$
P\left[\left|Y_{n}^{*}\right|>n^{-a}\right]=0
$$

Also Theorem 4.1 gives

$$
\sup _{t}\left|P\left[Z_{n}^{*} \leq t\right]-\Phi(t)\right|=O\left(n^{-1 / 7}(\log n)^{3}\right) .
$$

Now applying the Lemma 3.1 we get

$$
\begin{gathered}
\sup _{t}\left|P\left[\frac{\hat{f}_{n}(x, y)-f(x, y)}{\sqrt{V} a r\left\{\hat{f}_{n}(x, y)\right\}} \leq t\right]-\phi(t)\right| \\
=O\left(n^{-1 / 7}(\log n)^{3}+n^{-a}\right) .
\end{gathered}
$$

In particular for $a=\frac{1}{10}-\tau, 0<\tau<\frac{1}{10}$ and $\alpha=\frac{1}{5}$ we get the right hand side of (4.9) as $O\left(n^{-\left(\frac{1}{10}-\tau\right)}\right)$ and hence the theorem. 


\section{Speed of Convergence to Asymptotic Normality of the Estimated Tran- sition Density}

If $f(x, y)$ be the stationary p.d.f. for $\left(X_{j}, X_{j+1}\right)$ then $f(y / x)=f(x, y) / f(x)$ is the transition p.d.f. of $X_{j+1}$ given $X_{j}=x$. Let $I$ be an interval on $R$ and $F_{0}$ be the class of all distributions having the density ' $f$ ' such that, $0<\varepsilon_{1} \leq \inf _{x \in I} f(x) \leq \sup _{x \in I} f(x) \leq \varepsilon_{2}$.

Further we assume, in this section, $K$ is differentiable.

A logical estimator for the transition density is, therefore,

$$
\hat{f}_{n}(y / x)=\hat{f}_{n}(x, y) / \hat{f}_{n}(x)
$$

where $\hat{f}_{n}(x, y)$ and $\hat{f}_{n}(x)$ are as in (4.1) and (2.2) respectively. Under some assumptions Yakowitz (1985) proved that,

$$
\left(n h^{2}\right)\left[\hat{f}_{n}(y / x)-f(y / x)\right]^{d} \sim\left(0, f(x, y)\left[\int K^{2}(\alpha, \beta) d \alpha d \beta\right] / f^{2}(x)\right)
$$

Now, $\left(n h^{2}\right)^{1 / 2}\left[\hat{f}_{n}(y / x)-f(y / x)\right]$

$$
\begin{aligned}
& =\left(n h^{2}\right)^{1 / 2}\left[\frac{\hat{f}_{n}(x, y) / f(x)}{\hat{f}_{n}(x) / f(x)}-\frac{f(x, y)}{f(x)}\right] \\
& =\left(n h^{2}\right)^{1 / 2}\left[\hat{f}_{n}(x, y) / f(x)-f(y / x) \cdot \hat{f}_{n}(x) / f(x)\right] /\left[\hat{f}_{n}(x) / f(x)\right] \\
& =W_{n} / T_{n}, \text { say }
\end{aligned}
$$

where

$$
\begin{aligned}
W_{n}= & \left(n h^{2}\right)^{1 / 2}\left[\hat{f}_{n}(x, y) / f(x)-f(y / x) \hat{f}_{n}(x) / f(x)\right] \\
= & Z_{n}+Y_{n} \\
Z_{n} & =\left(n h^{2}\right)^{1 / 2}\left[\hat{f}_{n}(x, y)-f(x, y)\right] / f(x) \\
Y_{n} & =-\left(n h^{2}\right)^{1 / 2} f(y \mid x)\left[\hat{f}_{n}(x)-f(x)\right] / f(x)
\end{aligned}
$$

and

$$
T_{n}=\hat{f}_{n}(x) / f(x) .
$$

Also we have seen in section 4 that

$$
\begin{aligned}
\sup _{t}\left|P\left[Z_{n} \leq t\right]-\Phi(t)\right| & =O\left(n^{-\left(\frac{1}{10}-\tau\right)}\right) \\
& =O\left(b_{n}\right), \text { say. }
\end{aligned}
$$

Under the above respresentation we have the following results :

Result 5.1. For any $a_{n}>0, \delta>0$ and $C_{1}>0$,

$$
P\left[\left|Y_{n}\right|>a_{n}\right] \leq 2 . \exp \left[-\delta\left(A a_{n}-C_{1} h^{2} \sqrt{n h^{2}}\right)^{2} /\left(A_{1}^{2} h^{2}\right)\right]
$$

if $A=f(x) / f(y / x)<\infty$ and $O<A_{1}=K(x)<\infty$.

Result 5.2. For any $\eta>0, \delta>0$ and $C_{1}>0$,

$$
P\left[\left|T_{n}-1\right|>\eta\right] \leq 2 . \exp \left[-n \delta\left(\eta f(x)-C_{1} h^{2}\right)^{2} / A_{1}^{2}\right]
$$

where $0<A_{1}=K(x)<\infty$. 
Before proving the above two results we state and prove the following theorem :

THeorem 5.1. For a stationary Markov $G_{2}$-sequence $\left\{X_{i}\right\}$ satisfying (1.1), if $F_{n}(y)$ and $F(y)$ are the empirical and true distribution functions respectively then for any $d>0$ and $\delta>0$,

$$
\sup _{y} P\left[\left|F_{n}(y)-F(y)\right| \geq d\right]<<2 \exp \left(-n d^{2} \delta\right)
$$

Proof. We have $F_{n}(y)-F(y)$

$$
\begin{aligned}
& =\left[\sum_{i=1}^{n} I_{y}\left(X_{i}\right)-E \sum_{1}^{n} I_{y}\left(X_{i}\right)\right] / n \text { where } I_{y}\left(X_{i}\right)=1 \text { if } y \geq X_{i} \\
& =0 \text { otherwise } \\
& =\frac{1}{n} \sum_{i=1}^{n} Z_{i} \text { where } Z_{i}=I_{y}\left(X_{i}\right)-E I_{y}\left(X_{i}\right) .
\end{aligned}
$$

Here $Z_{1}, Z_{2}, \ldots, Z_{n}$ are uniformly bounded random variables with $E\left(Z_{k}\right)=0, V\left(Z_{k}\right)=$ $E\left(Z_{k}^{2}\right) \leq \rho^{2} \sigma^{2} \leq F(y)(1-F(y)) \leq 1 / 4$ and $\left|Z_{k}\right| \leq 1$.

Let $Z=\sum_{1}^{n} Z_{i}$. Therefore $F_{n}(y)-F(y)=Z / n$.

Now

$$
\begin{aligned}
E\left(e^{t Z_{k}}\right) & =E\left[1+t Z_{k}+\frac{t^{2} Z_{k}^{2}}{2}+\frac{t^{3} Z_{k}^{3}}{3 !}+\frac{t^{4} Z_{k}^{4}}{4 !}+\ldots\right] \\
& \leq 1+\frac{t^{2}}{2} E Z_{k}^{2}+E\left[\frac{t^{3}}{3 !} Z_{k}^{2}+\frac{t^{4}}{4 !} Z_{k}^{2}+\ldots\right] \\
& \leq 1+\frac{t^{2} \rho^{2} \sigma^{2}}{2}+\rho^{2} \sigma^{2}\left[\frac{t^{3}}{3 !}+\frac{t^{4}}{4 !} \ldots\right] \quad \text { (because of } G_{2}-\text { property) } \\
& =1+\rho^{2} \sigma^{2} t^{2} / 2+\rho^{2} \sigma^{2} \sum_{n=3}^{\infty} t^{n} / n ! \\
& =1+\rho^{2} \sigma^{2} \frac{t^{2}}{2}+\rho^{2} \sigma^{2} t^{2} \sum_{n=3}^{\infty} \frac{t^{n-2}}{n !} \\
& =1+\left(\rho^{2} \sigma^{2} t^{2} / 2\right)+\frac{\rho^{2} \sigma^{2} t^{2}}{6} \sum_{n=3}^{\infty} \frac{t^{n-2}}{(n-3) !} \quad\left(\text { since } \frac{1}{n !} \leq \frac{1}{6} \frac{1}{(n-3) !} \text { for } n \geq 3\right) \\
& =1+\left(\rho^{2} \sigma^{2} t^{2} / 2\right)\left\{1+t e^{t} / 3\right\} \\
& \leq \exp \left\{\frac{1}{2} \rho^{2} \sigma^{2} t^{2}\left(1+t e^{t} / 3\right)\right\} \quad\left(\text { since } 1+x \leq e^{x} \quad \text { for } x \geq 0\right) \\
& \leq \exp \left\{\frac{1}{8} \rho^{2} t^{2}\left(1+t e^{t} / 3\right)\right\} .
\end{aligned}
$$

Again from Rosenblatt (1970) we have,

$\left|E\left(\pi_{1}^{n} e^{t Z_{i}}\right)-\pi_{1}^{n} E\left(e^{t Z_{i}}\right)\right| \leq(n-1) M \rho^{n}$ where $M$ is a constant independent of $y$ and $O<\rho<1$. This implies

$$
-(n-1) M \rho^{n}+\prod_{i=1}^{n} E\left(e^{t Z_{i}}\right) \leq M(t) \leq(n-1) M \rho^{n}+\prod_{i=1}^{n} E\left(e^{t Z_{i}}\right)
$$


where $M(t)=E\left(e^{t Z}\right)=E\left(e^{t \Sigma Z_{i}}\right)=E\left(\stackrel{\pi}{n}_{1}^{n} e^{t Z_{i}}\right)$.

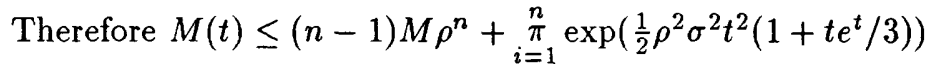

$$
\begin{aligned}
& <<2 \cdot \exp \left\{\frac{n}{2} \rho^{2} \sigma^{2} t^{2}\left(1+t e^{t} / 3\right)\right\} \\
= & 2 \cdot e^{n c}, \text { where } c=\frac{1}{2} \rho^{2} \sigma^{2} t^{2}\left(1+t e^{t} / 3\right) \\
\leq & e^{2 n c}, \text { because } 2<e<3 \\
\leq & \exp \cdot\left\{2 \cdot n \cdot \frac{1}{2} \rho^{2} \sigma^{2} t^{2}\left(1+t e^{t} / 3\right)\right\} \\
\leq & \exp \left\{\frac{n}{4} t^{2} \rho^{2}\left(1+t e^{t} / 3\right)\right\} \\
= & \exp \left\{\frac{1}{2} t^{2} D^{2}\left(1+t e^{t} / 3\right)\right\}, \text { where } D^{2}=\frac{n}{2} \rho^{2} .
\end{aligned}
$$

Also we have the inequality (see Renyi p. 384)

$$
P\left[Z \geq M+\frac{T+\log M(t)}{t}\right] \leq e^{-T}
$$

where $M$ is the expectation of $Z$.

Here $M=0$ and therefore

$$
P\left(Z \geq\left\{T+\frac{1}{2} t^{2} D^{2}\left(1+t e^{t} / 3\right)\right\} / t\right) \leq e^{-T} .
$$

Put $t=\sqrt{2 T} / D$ and therefore

$$
P\left(Z \geq D \sqrt{(2 T)}\left\{1+\frac{\sqrt{(2 T)}}{6 D} e^{\sqrt{(2 T)} / D}\right\}\right) \leq e^{-T} .
$$

Now substitution of $\lambda=\sqrt{(2 T)}$ gives

$$
P\left(Z \geq \lambda D\left\{1+(\lambda / 6 D) e^{\lambda / D}\right\}\right) \leq e^{-\lambda^{2} / 2} .
$$

Applying the obtained result to $-Z$, we find that

$$
P\left(|Z| \geq \lambda D\left\{1+(\lambda+6 D) e^{\lambda / D}\right\}\right) \leq 2 e^{-\lambda^{2} / 2} .
$$

In order to write this in a more convient form, we restrict ourselves to the case $\frac{\lambda}{D} \leq 1$. We have then

$$
e^{\lambda / D} \leq e<3
$$

Now we put $\gamma=\lambda(1+\lambda / 2 D)$ and then $\lambda \leq \gamma \leq \lambda(1+\gamma / 2 D)$.

$$
P(|Z| \geq \gamma D) \leq 2 e^{-\frac{\gamma}{2}(1+\gamma / 2 D)^{-2}}
$$

i.e.

$$
P\left(\left|F_{n}(y)-F(y)\right| \geq \frac{\gamma D}{n}\right) \leq 2 e^{-\left\{\gamma^{2} / 2(1+\gamma / 2 D)^{-2}\right\}} \text { for all } y
$$


i.e.

$$
P\left(\left|F_{n}(y)-F(y)\right| \geq d\right) \leq 2 e^{-\left\{n^{2} d^{2} / 2 D^{2}\left(1+\frac{n d}{2 D^{2}}\right)^{-2}\right\}} \text { for all } y .
$$

Now replacing $D^{2}$ by $\frac{n}{2} \rho^{2}$ we have

$$
\begin{aligned}
P\left(\left|F_{n}(y)-F(y)\right| \geq d\right) & \leq 2 \exp \left(-n \rho^{2} d^{2} /\left(d+\rho^{2}\right)^{2}\right) \\
& \leq 2 \exp \left(-n d^{2} \delta\right) .
\end{aligned}
$$

Since all the upper bounds in our calculations are independent of $y$,

$$
\sup _{y} P\left[\left|F_{n}(y)-F(y)\right| \geq d\right]<<2 \exp \left(-n d^{2} \delta\right) .
$$

REMARK. The result in this theorem perhaps follows from P.K. Bhattacharyya (1972) but for completeness and independent interest we have given some details.

Proof of Result 5.1.

$$
\begin{aligned}
P\left[\left|Y_{n}\right|>a_{n}\right] & =P\left[\left(n h^{2}\right)^{1 / 2} / f(x) f(y / x)\left|\hat{f}_{n}(x)-f(x)\right|>a_{n}\right] \\
& =P\left[\left(n h^{2}\right)^{1 / 2}\left|\hat{f}_{n}(x)-f(x)\right|>\varepsilon\right], \text { where } \varepsilon=A . a_{n} \\
& \leq P\left[\left(n h^{2}\right)^{1 / 2}\left\{\left|\hat{f}_{n}(x)-E \hat{f}_{n}(x)\right|+\left|E \hat{f}_{n}(x)-f(x)\right|\right\}>\varepsilon\right] .
\end{aligned}
$$

Now,

$$
\begin{aligned}
E\left(\hat{f}_{n}(x)\right)-f(x) & =O\left(h^{2}\right), \text { as in }(4.4) \\
& \leq C_{1} h^{2} .
\end{aligned}
$$

Therefore,

$$
\begin{aligned}
P\left[\left|Y_{n}\right|>a_{n}\right] & \leq P\left[\left|\hat{f}_{n}(x)-E \hat{f}_{n}(x)\right|>\left\{\varepsilon /\left(n h^{2}\right)^{1 / 2}-C_{1} h^{2}\right\}\right] \\
& =P\left[\left|\hat{f}_{n}(x)-E \hat{f}_{n}(x)\right|>\left\{\varepsilon^{\prime} /\left(n h^{2}\right)^{1 / 2}\right\}\right]
\end{aligned}
$$

where

$$
\begin{aligned}
\varepsilon^{\prime} & =\varepsilon-C_{1} h^{2}\left(n h^{2}\right)^{1 / 2} \\
& =A \cdot a_{n}-C_{1} h^{2}\left(n h^{2}\right)^{1 / 2} \\
& \leq \sup _{y} P\left[\left|F_{n}(y)-F(y)\left\|\mid \frac{d}{d y} K((x-y) / h) \frac{1}{h}\right\|_{1}>\varepsilon^{\prime} /\left(n h^{2}\right)^{1 / 2}\right\}\right] \\
& \quad \text { (integrating by parts). }
\end{aligned}
$$

Using (2.4), $\left\|\frac{d}{d y} K((x-y) / h) \frac{1}{h}\right\|_{1}=\int_{-\infty}^{\infty} \frac{d}{d y} K((x-y) / h) \frac{1}{h} d y=A_{1}$.

From (5.10) we have,

$$
\begin{aligned}
P\left[\left|Y_{n}\right|>a_{n}\right] & \leq \sup _{y} P\left[\left|F_{n}(y)-F(y)\right|>\left\{\varepsilon^{\prime} / A_{1}\left(n h^{2}\right)^{1 / 2}\right\}\right] \\
& \leq 2 \cdot \exp \left[-n \delta\left\{\varepsilon^{\prime^{2}} /\left(A_{1}^{2} n h^{2}\right)\right\}\right] \\
& =2 \cdot \exp \left[-\delta\left\{A a_{n}-C_{1} h^{2}\left(n h^{2}\right)^{1 / 2}\right\}^{2} /\left(h^{2} A_{1}^{2}\right)\right] .
\end{aligned}
$$


Proof of RESULT 5.2. Following the proof of Result 5.1 we have,

$$
\begin{aligned}
P\left[\left|T_{n}-1\right|>\eta\right] \leq & P\left[\left|\hat{f}_{n}(x)-f(x)\right|>\eta f(x)\right] \\
& =P\left[\left|\hat{f}_{n}(x)-f(x)\right|>\eta_{1}\right] \text { where } \eta_{1}=\eta . f(x) \\
& \leq P\left[\left|\hat{f}_{n}(x)-E \hat{f}_{n}(x)\right|>\eta_{1}-C_{1} h^{2}\right] \\
& \leq \sup _{y} P\left[\left|\hat{F}_{n}(y)-F(y)\right| \geq\left(\eta_{1}^{\prime} / A_{1}\right)\right] \\
& \text { where } \eta_{1}^{\prime}=\eta_{1}-C_{1} h^{2} \\
& =2 \cdot \exp \left[-n{\eta_{1}^{\prime}}^{2} \delta / A_{1}^{2}\right] \\
& =2 \cdot \exp \left[-n \delta\left(\eta_{1}-C_{1} h^{2}\right)^{2} / A_{1}^{2}\right] \\
& =2 \cdot \exp \left[-n \delta\left(\eta f(x)-C_{1} h^{2}\right)^{2} / A_{1}^{2}\right]
\end{aligned}
$$

Now using (5.7), (5.8) and Lemma 3.1 we have the following result.

RESUlT 5.3 .

$$
\begin{gathered}
\sup _{t}\left|P\left[W_{n} \leq t\right]-\Phi(t)\right|=O\left(b_{n}\right)+O\left(a_{n}\right)+P\left[\left|Y_{n}\right|>a_{n}\right] \\
<<n^{-\left(\frac{1}{10}-\tau\right)}+a_{n}+\exp \left[-\delta\left(A a_{n}-C_{1} h^{2} \sqrt{\left(n h^{2}\right)}\right)^{2} /\left(A_{1}^{2} h^{2}\right)\right]
\end{gathered}
$$

where $h=O\left(n^{-\frac{1}{5}}\right)$.

Let us state another result which is due to Michel and Pfanzagl (1971).

Result 5.4. Let $(\Omega, \mathcal{F}, P)$ be a probability space and $W_{n}, T_{n}$ be $\mathcal{F}$-measurable functions. Then, for any $\eta>0$,

$$
\sup _{t}\left|P\left(W_{n} / T_{n}\right) \leq t-\Phi(t)\right| \leq \sup _{y}\left|P\left(W_{n} \leq y\right]-\Phi(y)\right|+P\left[\left|T_{n}-1\right|>\eta\right]+\eta .
$$

where $\Phi(\cdot)$ is the standard normal distribution function.

Now we have the following theorem :

THEOREM 5.2. If $\Phi$ be the distribution function of a standard normal distribution then under the assumption of Theorem 4.2

$$
\left.\sup _{F_{0}} \sup _{x \in I} \sup _{n \geq n_{0}} \sup _{t} \mid P\left[\left\{\hat{f}_{n}(y / x)-f(y / x)\right\} /\left(V\left(\hat{f}_{n}(y / x)\right)\right)^{1 / 2}\right\} \geq t\right]-\Phi(t) \mid<<n^{-\frac{1}{10}+\tau} .
$$

Proof. Applying Result 5.2 and Result 5.3 in Result 5.4 we have,

$$
\begin{gathered}
\sup _{t}\left|P\left[\left(W_{n} / T_{n}\right) \leq t\right]-\Phi(t)\right| \\
<<n^{-\frac{1}{10}+\tau}+a_{n}+\exp \left[-\delta\left(A a_{n}-C_{1} h^{2} \sqrt{\left(n h^{2}\right)}\right)^{2} / h^{2} A_{1}^{2}\right] \\
+\exp \left\{-n \delta\left(\eta f(x)-C_{1} h^{2}\right)^{2} / A_{1}^{2}\right\}+\eta .
\end{gathered}
$$

Putting $a_{n}=0\left(n^{-\frac{1}{5}}(\log n)^{1 / 2}\right)$ and $\left.\eta=0((\log n) / n)^{1 / 2}\right)$, we get

$$
\begin{gathered}
\left.\sup _{t} \mid P\left\{\left(\hat{f}_{n}(y / x)-f(y / x)\right) /\left(V\left(\hat{f}_{n}(y / x)\right)\right)^{1 / 2}\right\} \leq t\right]-\Phi(t) \mid \\
<<n^{-\frac{1}{10}+\tau}+n^{-\frac{1}{5}}(\log n)^{1 / 2}+\exp \left(-\delta\left(A a_{n}-C_{1} h^{2} \sqrt{\left(n h^{2}\right)}\right)^{2} / h^{2} A_{1}^{2}\right) \\
+\exp \left(-n \delta\left(\eta f(x)-C_{1} h^{2}\right)^{2} A_{1}^{2}\right)+((\log n) / n)^{1 / 2}<<n^{-\frac{1}{10}+\tau} .
\end{gathered}
$$




\section{Concluding Remarks}

Prakasa Rao (1977) considered the problem of estimating the unknown density under much stronger condition $D_{0}$ and got the result $O\left(n^{-\frac{1}{3}+\tau}\right), 0<\tau<\frac{1}{3}$. Neither the problem of estimating the stationary density nor the transition density has been considered by him. Although our rate result is poorer than Prakasa Rao (1977), yet our results have some theoretical interest in the sense that there is no work in the available literature regarding the rate of convergence to normality for estimating the stationary density and transition density under much weaker assumption $G_{2}$ which has so much applications in time series data.

Also, in this context, one thing should be noted is that we have taken the windowwidth $h_{n}$ as $O\left(n^{-\frac{1}{5}}\right)$ which is optimal in the sense that it minimizes mean-integrated square error and this choice of window-width is different from that of Prakasa Rao (1977) where he took the window-width as $O\left(n^{-\frac{1}{3}}\right)$.

In Theorem 4.2 and Theorem 5.2 we conjecture that the rates should be $O\left(n^{-\frac{1}{7}}(\log n)^{3}\right)$. Actually in our earlier version we obtained the above rate but unfortunately there was a technical flaw. Thanks to the referee for pointing out this.

\section{References}

Basu, A.K. and Sahoo, D.K. (1989) : Berry-Esséen type of theorems for sequential density estimation. Sequential Analysis, 119-134.

Basu, A.K. and Sahoo, D.K. (1989) : Rate of convergence for nonparametric density estimation in linear process. Bull of Inform. and Cybernatics, 23, 209-224.

Bhattacharya, P.K. (1972) : Probabilities of large deviations of sums of random variables. Sankhy $\bar{a}$, Ser. A, 34, 9-16.

Doob, J.L. (1953) : Stochastic processes, Wiley, Newyork.

Michel and Pfanzagl (1971) : The accuracy of the normal approximation for minimum contrast estimates, Z. Wahrs Verw. Gebiete, 18, 73-84.

Philipp (1969) : The remainder in the central limit theorem for mixing stochastic processes. Ann. Math. Statist., 40, 601-609.

Prakasa Rao, B.L.S. (1977) : Berry-Esséen bound for density estimators of stationaryMarkov processes. Bull. Math. Statist., 17, 15-21.

Renyi (1970) : Probability theory, North Holland Publishing Co.

Rosenblatt, M. (1970) : Density estimates and Markov sequences. Nonparametric techniques in statistical inference, ed. M. Puri, Oxford - Cambridge Univ. Press.

Rosenblatt, M. (1971) : Curve estimates, Ann. Math. Statist., 42, 1815-1842.

Roussas (1967) : Non-parametric estimation in Markov processes, Technical Report No. 110, Dept. of Statistics, Univ. of Wisconsin, Madison, Wisconsin.

Roussas (1969) : Non-parametric estimation of the transition distriibution function of a Markov process : Ann. Math. Statist., 40, 1386-1400.

Yakowitz (1985) : Non-parametric estimation, prediction and regression for Markov sequences : Journal of the Americal Statistical Association, 80, 215-221. 
Yakowitz (1989) : Nonparametric density and regression estimation for Markov sequences without mixing assumptions, J. Mult. Analysis., 30, 124-136.

Received April 2, 1996

Revised December 13, 1996

Re-revised January 6, 1998 